\section{More need for the "old" in new public health research in the Americas}

Martha Pelaez 1

1 Pan American Health Organization, Washington, D.C., United States of America.
Research on the health of older adults in Latin America and the Caribbean has been neglected in epidemiology and public health. This is of grave concern since dramatic changes in fertility and mortality rates in recent decades ensure a rapid aging of the population in the Western Hemisphere. Persons 60 and older in Latin America and the Caribbean now make up 8\% of the total population; by the middle of this century that figure will grow to $22 \%$. In absolute numbers, the population of older persons will total over 180 million by 2050. In the coming decades we expect to find poorer health status and greater disability in the cohorts of persons who survived childhood and adult diseases. That higher survivial rate is due more to public health interventions and less to an improvement in their socioeconomic status. Therefore, policymakers in Latin America and the Caribbean need to understand the correlations among different gradients of poverty, access to health care, social networks, and health in old age.

The epidemiology of aging and longevity provides a framework for understanding both the risk factors associated with old age and the atypical manifestations of infectious diseases in persons with diminished immunity. Elders in Latin America and the Caribbean will often suffer from a complex mix of infectious and noncommunicable diseases, and infectious diseases will continue to be an important cause of adult mortality. Thus, we need more information on the complex interaction of diseases, frailty, socioeconomics, and social networks in old age. We still have great gaps in our knowledge of aging in the Region of the Americas; it is not surprising that older persons are underrepresented as a target population in the public health literature.

The essential functions of public health, including monitoring, evaluation, and analysis of the health status of older persons, require the development and use of multidisciplinary research designs (1), new assessment protocols, and adequate survey instruments in order to capture the specific characteristics of older adults. With the support of the National Institute on Aging of the United States of America, the Pan American Health Organization is collaborating with researchers in the Americas to develop more efficient data collection instruments and thus promote demographic and epidemiological studies of older persons. Ideally, in the future, information systems and researchers in different countries will collect comparable data on health and aging and therefore produce important insights into the roles that culture, socioeconomic status, and different institutional approaches play in the health of older persons.

While aging research in the developed countries has produced a vast scientific literature, much more dialogue among researchers in all the countries of the Americas is needed in order to develop a better understanding of the health and well-being of older adults. In this issue of the Revista Panamericana de Salud Pública/Pan American Journal of Public Health we find three important contributions to this dialogue: Lima-Costa et al. on socioeconomic position and health in a population of Brazilian elderly (2), Rozenfeld et al. on medication as a risk factor for falls in older women in Brazil (3), and García-Rivera and Rigau-Pérez on dengue severity in the elderly in Puerto Rico (4). The themes highlighted by each of these articles present important components of an agenda for research on aging. 


\section{Socioeconomic position and health}

Lima-Costa et al. show a strong relationship between gradients of poverty and poorer self-reported health as well as worse physical functioning. They demonstrate that "a small difference in monthly family income was sufficiently sensitive to identify elders in worse health condition, even within a community that appears to be uniformly poor" (2). In arriving at this conclusion, they utilized not only self-rated health but also measures of physical functioning. While these authors say their findings are different from those established in studies in some developed countries, some recent research in the United States of America also tends to support a strong relationship between income and physical function and disability (5). Similar results have come from a study called Health, Well-Being and Aging in Latin America and the Caribbean (the "SABE Project"). That survey study, which collected data on socioeconomic status, health, and function among older persons living in seven large cities in the Americas, found that the socioeconomic gradients were notable predictors of poor health and disability in all cities, with the exception of Havana, where the measure of income was less significant. Lima-Costa et al. (2) also explore whether health differentials are related only to family income, and they find that income is also related to access to medical care and use of medications. If the number of older persons living in poverty continues to increase, the findings in these studies translate into strong effects on the health, functioning, and disability of elders in developing countries. To develop policies that can effectively ameliorate inequalities in health, policymakers will need to address a wide range of determinants, including access to medications, proper nutrition, social services, and better income security in old age.

\section{Misuse of medications as a risk factor for falls}

Compared with other age groups, older persons have a higher rate of falls and, consequently, a higher use of health services. Falls are often associated with frailty, so Rozenfeld et al. (3) made a very interesting selection for their study of falls by focusing on nonfrail women participating in a university-sponsored program that provides educational, cultural, and medical care activities for older women in Rio de Janeiro. The association between medication use and the prevalence of falls is well documented in the scientific literature (6); however, the situation as presented by Rozenfeld et al. (3) points to a major public health issue regarding the high number of medications used by older women without close monitoring by a health care provider. The findings of this study are particularly important because so little is known about access to, use of, and regular monitoring of medication among older adults. The SABE survey found that more than $50 \%$ of older persons regularly take three or more medications and that $80 \%$ of the cost of medications is paid by either the older person or a family member. In countries where medications are obtained with little regulatory control, it is estimated that the type of medication taken by the individual as well as adherence to the therapeutic regime for each medication will vary significantly with the individual's ability to pay. Therefore, it is reasonable to expect that misuse of medication may play an even more important role in falls among older women throughout the Region of the Americas. The article by Rozenfeld et al. (3) points to the need for more studies to address a number of concerns, including: What elements of cardiovascular programs are effective in preventing falls? Does treatment of visual problems help to prevent falls? What is the cost-effectiveness of subsidized mental health treatment programs for older persons? What elements of exercise programs are effective in reducing the use of anxiolytics/sedatives and other psychoactive agents? 


\section{Dengue severity in the elderly}

Another important contribution to aging research in this issue of the Revista/Journal is the paper by García-Rivera et al. on dengue severity in the elderly in Puerto Rico (4). The relationship between immune senescence and the clinical manifestations of infectious diseases has not been the subject of enough studies in the Region of the Americas. García-Rivera et al. (4) make a good case for expanding aging research to include studies on the atypical presentations and complications of infectious diseases in old age as well as in building a body of knowledge on the interaction of infectious diseases with chronic diseases. Additionally, this article alerts us to the importance of including older adults in the surveillance and study of other infectious diseases that are reappearing in the world.

It is clear that studies on the health of older persons are relatively underrepresented in the public health programs of most countries. There is a virtual absence of data on morbidity and disability levels among the aged. Such information is critical for assessing the magnitude and nature of inequalities in health and the efficient targeting of preventive and curative interventions for older persons. The papers in this issue of the Revista/Journal will generate discussion, and in the coming months we hope to see more articles on this vital issue of aging in the Americas.

\section{REFERENCES}

1. Pan American Health Organization. Public health in the Americas: conceptual renewal, performance assessment, and bases for action. Washington, D.C.: PAHO; 2002.

2. Lima-Costa MF, Barreto SM, Firmo JOA, Uchoa E. Socioeconomic position and health in a population of Brazilian elderly: the Bambuí Health and Aging Study (BHAS). Rev Panam Salud Publica 2003;13(6):387-394.

3. Rozenfeld S, Camacho LAB, Veras RP. Medication as a risk factor for falls in older women in Brazil. Rev Panam Salud Publica 2003;13(6):369-375.

4. García-Rivera EJ, Rigau-Pérez JG. Dengue severity in the elderly in Puerto Rico. Rev Panam Salud Publica 2003;13(6):362-368.

5. Kaplan GA. Behavioral, social, and socioenvironmental factors adding years to life and life to years. In: Hickey T, Speers M, Prohaska TR, eds. Public health and aging. Baltimore, Maryland: The Johns Hopkins University Press; 1997. Pp. 37-54.

6. Leipzig RM, Cumming RG, Tinetti ME. Drugs and falls in older people: a systematic review and meta-analysis: I. Psychotropic drugs. J Am Geriatr Soc 1999;47(1):30-39. 